\title{
MULTI-ANTENNA OBSERVATIONS IN THE LOW-FREQUENCY RADIO ASTRONOMY OF SOLAR SYSTEM OBJECTS AND RELATED TOPICS STUDIES
}

\author{
A. Konovalenko*, P. Zarka ${ }^{\dagger}$, H.O. Rucker ${ }^{\ddagger}$, V. Zakharenko*, \\ O. Ulyanov*, M. Sidorchuk*, S. Stepkin*, V. Melnik*, \\ N. Kalinichenko*, A. Stanislavsky*, P. Tokarsky*, V. Koliadin*, \\ V. Shepelev*, V. Dorovskyy*, I. Bubnov*, S. Yerin*, \\ A. Reznichenko*, G. Litvinenko*, N. Shevchuk*, A. Koval*, \\ I. Vasylieva*, K. Mylostna*, A. Skoryk* , A. Shevtsova*, \\ Y. Volvach*, E. Vasylkovsky*, V. Ryabov ${ }^{\S}$, A. Lecacheux ${ }^{\dagger}$, \\ L. Denis ${ }^{\mathbb{I}}$ M. Panchenko", G. Fischer", M. Imai**, \\ J.-M. Grießmeier ${ }^{\dagger \dagger}$, G. Mann ${ }^{\ddagger}$, O. Litvinenko*, A. Brazhenko ${ }^{\S}$, \\ R. Vashchishin ${ }^{\S}$, A. Frantsuzenko ${ }^{\S}$, V. Koshovy $\mathbb{I}$,

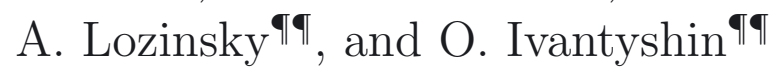

\begin{abstract}
Rapid progress currently takes place in the field of low-frequency radio astronomy in the meter-decameter-hectometer range of wavelengths. It is caused by a radical modernization of the existing radio telescopes, creation of a new generation of instruments, space-borne observations, and by the development of research on all classes of astrophysical objects, including the Solar System. On the other hand, a range of difficulties specific to low-frequency radio astronomy is known, which are caused by technical, methodological, and physical limitations. An effective strategy

\footnotetext{
* Institute of Radio Astronomy, National Academy of Sciences of Ukraine, Kharkov, Ukraine

$\dagger$ LESIA, Observatoire de Paris, CNRS, PSL/SU/UPMC/UPD/SPC, Meudon, France

$\ddagger$ Commission for Astronomy, Austrian Academy of Sciences, Graz, Austria

$\S$ Future University of Hakodate, Hakodate, Japan

I 3USN (Unité Scientifique de la Station de Nançay), Nançay, France

\| Space Research Institute, Austrian Academy of Sciences, Graz, Austria

** Department of Physics and Astronomy, University of Iowa, Iowa City, IA, USA

†† LPC2E - University of Orléans, Orléans, France

¥ Leibniz-Institut für Astrophysik Potsdam, Potsdam, Germany

$\S$ Poltava Gravimetrical Observatory of Institute of Geophysics, National Academy of Sciences
}

II Karpenko Physiko-Mechanical Institute, National Acad. of Sciences of Ukraine, Lviv, Ukraine
\end{abstract}


for overcoming these difficulties is based on synchronous observations using several radio telescopes separated by distances from a few to several thousand kilometers. This provides an opportunity to reduce and identify radio interference and the influence of the propagation media, to increase the sensitivity and resolution, and to solve many problems with higher efficiency. In recent years such simultaneous observations were carried out for the Sun, Jupiter, Saturn, interplanetary medium, pulsars, exoplanets, and transients using the radio telescopes UTR-2, URAN, GURT, NDA, NenuFAR, LOFAR and other. Parallel observations with the space missions WIND, STEREO, Cassini and Juno also facilitate improvement of the quality and reliability of low-frequency radio astronomical experiments.

\section{Introduction}

Low-frequency radio astronomy, especially at decameter wavelengths, differs radically in several respects from its higher frequencies counterparts. On one hand, radio emission at low frequencies carries essential new information about many astrophysical objects and processes in the universe. For this reason, low-frequency radio astronomy has been experiencing rapid growth all over the world during the last 2 decades (see Section 2). On the other hand, serious additional difficulties arise at low frequencies. These difficulties stem from much higher levels of terrestrial radio-frequency interference (RFI), severe distortion of the signals by propagation effects, and a range of other negative factors (see Section 3).

The central point of this paper is that most of these problems could be radically overcome only by synergy between several radio telescopes. By "synergy" we mean synchronous observations of the same objects by different radio telescopes, separated by distances up to several thousands kilometers and more. Both terrestrial and space-borne instruments may be used for such parallel observations with overlapping or even non-overlapping
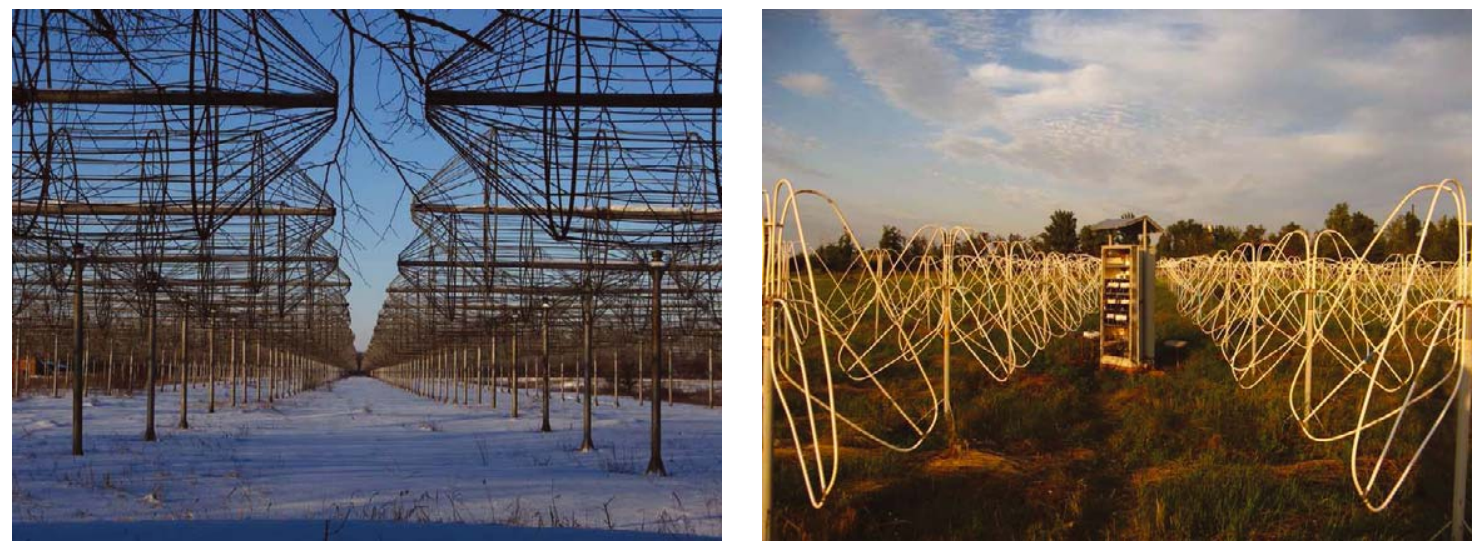

Figure 1: Radio telescopes at Kharkiv, Ukraine. Left: The UTR-2 radio telescope, $f=$ 8...32 MHz, $A_{\text {eff } \max }=150,000 \mathrm{~m}^{2}, \quad N=2040$. "North-South" arm: $1.8 \mathrm{~km} \times 60 \mathrm{~m}$. Right: Several sub-arrays $(5 \times 5$ elements each) of the new Giant Ukrainian Radio Telescope (GURT), $f=8 \ldots 80 \mathrm{MHz}$. 
(complementary) frequency ranges. Normally, neither modification of hardware/software subsystems nor mutual coherence between the instruments are required (except for the VLBI mode). Therefore, the idea of synergy may be quite easily implemented using the already existing radio telescopes.

We illustrate the idea with some results of our simultaneous observations of diverse classes of objects by the Ukrainian low-frequency radio telescopes (see Section 2) and the NDA radio telescope (Nançay, France), which are separated by distances from several to several thousand kilometers.

\section{Current status}

More than ten low-frequency radio telescopes are currently available worldwide. Several instruments have had operational history of many decades: UTR-2 (Ukraine, constructed in the beginning of 1970s, Figure 1) [Konovalenko et al., 2016], Nançay Decameter Array or NDA (France, constructed in the end of 1970s) [Boischot et al., 1980], four telescopes of the Ukrainian URAN network (Ukraine, constructed 1970s-1980s, Figure 2) [Konovalenko et al., 2016]. The parameters of the Ukrainian instruments and their back-end facilities used for registration of signals are summarized in Tables 1 and 2, respectively.
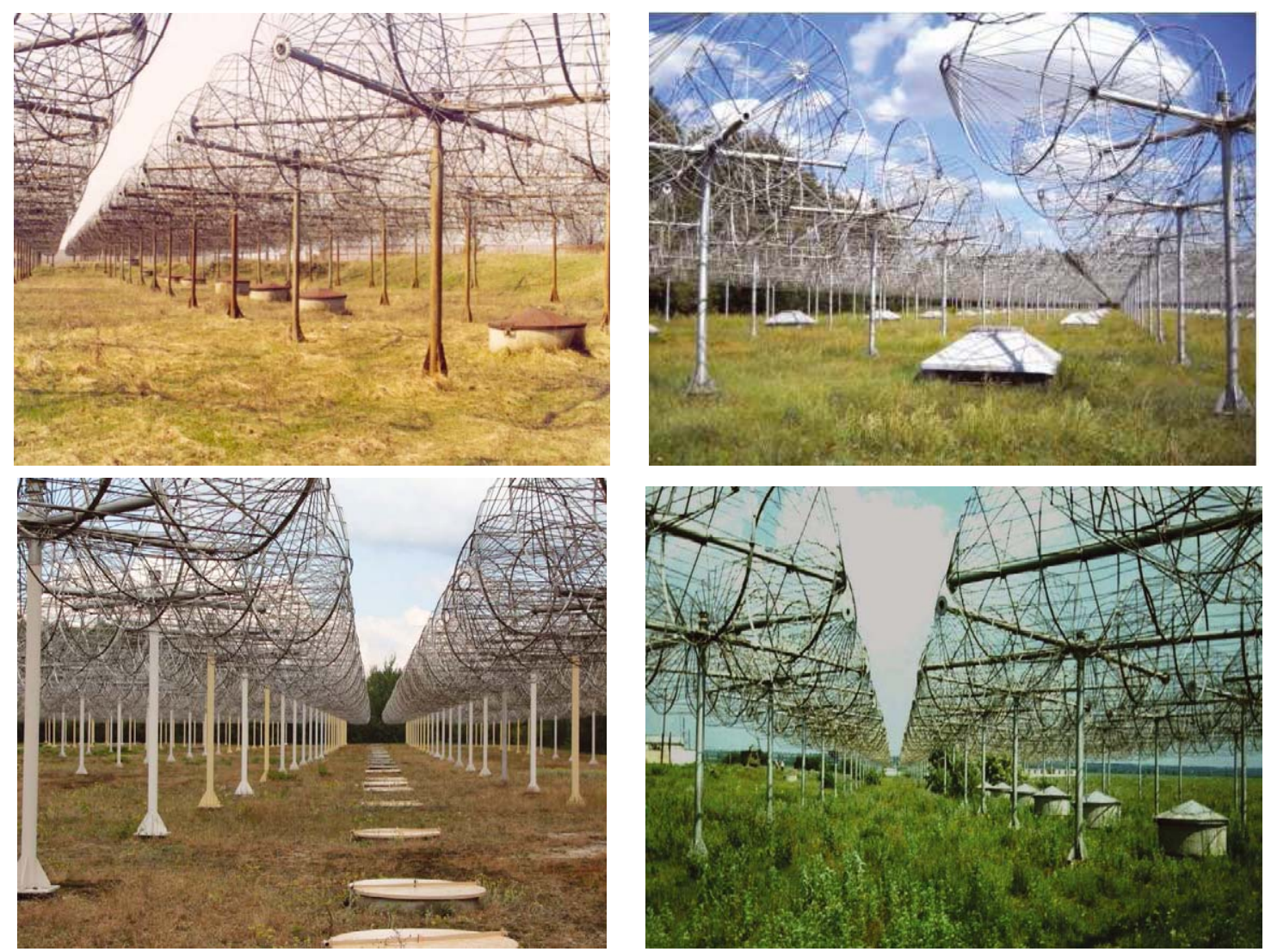

Figure 2: Ukrainian URAN-1...URAN-4 radio telescopes; $\sum N(U R A N-1 \ldots 4)=96 \times 2+512 \times 2+$ $256 \times 2+128 \times 2=1984 ; \sum N(U T R-2+U R A N-1 \ldots 4)=4024 ; A_{\text {eff total }} \approx 200,000 \mathrm{~m}^{2}$. 
Table 1: Main parameters of Ukrainian decameter radio telescopes.

\begin{tabular}{|c|c|c|c|c|c|c|}
\hline $\begin{array}{l}\text { Radio } \\
\text { telescope }\end{array}$ & Allocation & $\begin{array}{l}\text { Freq. } \\
\text { range, } \\
{[\mathrm{MHz}]}\end{array}$ & $\begin{array}{c}\text { Max. } \\
\text { effective } \\
\text { area, } \\
{\left[\mathrm{m}^{2}\right]}\end{array}$ & $\begin{array}{l}\text { Number } \\
\text { of elements, } \\
\text { polarization }\end{array}$ & $\begin{array}{c}\text { Distance } \\
\text { from UTR-2 } \\
\text { (LOFAR), } \\
{[\mathrm{km}]}\end{array}$ & $\begin{array}{l}\text { Synthesis } \\
\text { or VLBI } \\
\text { resolution } \\
\text { at } 25 \mathrm{MHz}\end{array}$ \\
\hline UTR-2 & $\begin{array}{l}\text { Kharkiv, } \\
\text { RI NASU }\end{array}$ & $8-32$ & 140,000 & $\begin{array}{l}2040, \\
1 \text { linear }\end{array}$ & $\begin{array}{c}0 \\
(\approx 2000)\end{array}$ & $25^{\prime} \times 25^{\prime}$ \\
\hline URAN-1 & $\begin{array}{l}\text { Zmiiv, } \\
\text { RI NASU }\end{array}$ & $8-32$ & 5,500 & $\begin{array}{l}96, \\
2 \text { linear }\end{array}$ & $\begin{array}{l}42 \\
(\approx 1900)\end{array}$ & $15 "$ \\
\hline URAN-2 & $\begin{array}{c}\text { Poltava } \\
\text { PGO } \\
\text { NASU }\end{array}$ & $8-32$ & 28,000 & $\begin{array}{l}512, \\
2 \text { linear }\end{array}$ & $\begin{array}{l}150 \\
(\approx 1800)\end{array}$ & $5 "$ \\
\hline URAN-3 & $\begin{array}{l}\text { Lviv, } \\
\text { PMI } \\
\text { NASU }\end{array}$ & $8-32$ & 14,000 & $\begin{array}{l}256, \\
2 \text { linear }\end{array}$ & $\begin{array}{l}915 \\
(\approx 1000)\end{array}$ & $1^{\prime \prime}$ \\
\hline URAN-4 & $\begin{array}{l}\text { Odesa, } \\
\text { RI NASU }\end{array}$ & $8-32$ & 7,300 & $\begin{array}{l}128, \\
2 \text { linear }\end{array}$ & $\begin{array}{c}613 \\
(\approx 1500)\end{array}$ & $1.3^{\prime \prime}$ \\
\hline $\begin{array}{c}\text { GURT, } \\
\text { single } \\
\text { sub-array }\end{array}$ & $\begin{array}{l}\text { Kharkiv, } \\
\text { RI NASU }\end{array}$ & $8-80$ & $\begin{array}{l}350 \\
(\mathrm{f}=40 \\
\mathrm{MHz})\end{array}$ & $\begin{array}{c}25(5 \times 5) \\
2 \text { linear }\end{array}$ & $\begin{array}{c}0 \\
(\approx 2000)\end{array}$ & - \\
\hline
\end{tabular}

There are also several new low-frequency radio telescopes that are either in the constructiontesting phase or have been put into operation recently: LOFAR (Low Frequency Array,

Table 2: New generation broad-band digital spectral processors for UTR-2, URAN, GURT.

\begin{tabular}{|l|c|c|}
\hline \multicolumn{1}{|c|}{ Parameters } & DSPZ - UTR-2 & ADR - GURT \\
\hline Frequency band [MHz] & 33 & 80 \\
\hline Number of freq. channels & 8,192 & 16,384 \\
\hline Frequency resolution [kHz] & 4 & 6 \\
\hline Time resolution [ms] & 0.5 & 2 \\
\hline ADC resolution [bits] & 16 & 16 \\
\hline Dynamic range [dB] & 90 & 90 \\
\hline Input channels & 2 & 2 \\
\hline \multicolumn{2}{|c|}{ On-line real-time possibilities: } \\
\hline Fast Fourier transform & yes & yes \\
\hline Wave-form (non limited resolutions) & yes & yes \\
\hline Auto- and complex cross-spectra & yes & yes \\
\hline Sum-subtraction mode & no & yes \\
\hline Signals normalization & no & yes \\
\hline Signals delay & no & yes \\
\hline
\end{tabular}


the Netherlands) [van Haarlem et al., 2013], NenuFAR (New Extension in Nançay Upgrading LOFAR, France) [Zarka et al., 2012], LWA (Long Wavelength Array, USA) [Taylor et al., 2012], GURT (Giant Ukrainian Radio Telescope, Ukraine) [Konovalenko et al., 2016]. Some other instruments operating at higher (but close) frequency ranges could be also involved in such parallel observations as well as space-borne instruments.

\section{Problems specific to low-frequency radio astronomy}

Low-frequency radio astronomy differs in several respects from the radio astronomy exploring higher frequencies; observations at low frequencies pose a range of serious problems. These difficulties and methods for their solutions are summarized in Table 3. Below we discuss mainly the difficulties originating from propagation effects and RFI.

Table 3: Difficulties of the decameter wavelengths radio astronomy.

\begin{tabular}{|c|c|}
\hline $\begin{array}{l}\text { Problems of low-frequency } \\
\text { radio astronomy }\end{array}$ & Possible solution \\
\hline $\begin{array}{l}\text { High temperature of the galactic } \\
\text { background }\end{array}$ & $\begin{array}{l}\text { High effective area }\left(10^{4} \ldots 10^{6} \mathrm{~m}^{2}\right) \\
\text { Multi-telescope observations }\end{array}$ \\
\hline $\begin{array}{l}\text { Ground-based interferences (natu- } \\
\text { ral, artificial, narrow-band, broad- } \\
\text { band) }\end{array}$ & $\begin{array}{l}\text { High dynamic range of the front-end } \\
\text { High dynamic range and resolutions back-end } \\
\text { Broad-band antenna } \\
\text { High directivity, low side lobes of antenna } \\
\text { Special processing (clean) } \\
\text { Space-borne instruments } \\
\text { Multi-telescope observations }\end{array}$ \\
\hline $\begin{array}{l}\text { Ionosphere influence (refraction, } \\
\text { scintillations, absorption) }\end{array}$ & $\begin{array}{l}\text { Large field of view (multi-beams) } \\
\text { Adaptive antenna } \\
\text { Special processing (clean) } \\
\text { Space-borne instruments } \\
\text { Multi-telescope observations }\end{array}$ \\
\hline $\begin{array}{l}\text { Low angular resolution in single- } \\
\text { dish mode (low } D / \lambda \text { ratio) }\end{array}$ & $\begin{array}{l}\text { VLBI (ground-ground; ground-space) } \\
\text { Multi-telescope observations }\end{array}$ \\
\hline
\end{tabular}

Analysis and interpretation of observations at low frequencies are severely impeded by propagation effects (scintillations, refraction). The passage of signals through interplanetary and ionospheric plasmas, which are essentially inhomogeneous and non-stationary, often causes severe distortion of the intrinsic dynamic spectra. The distortion manifests itself as deep modulation of the time-frequency power distribution by diverse patterns. This spurious modulation of dynamic spectra may be deep, e.g. downs by $10 \mathrm{~dB}$ (and more) and ups by $3-5 \mathrm{~dB}$ are quite common. The patterns often have complex structure, which resembles the effects of interest. Figure 3 shows a few examples. Displaying the actual diversity of such patterns (including very bizarre ones) would require tens 

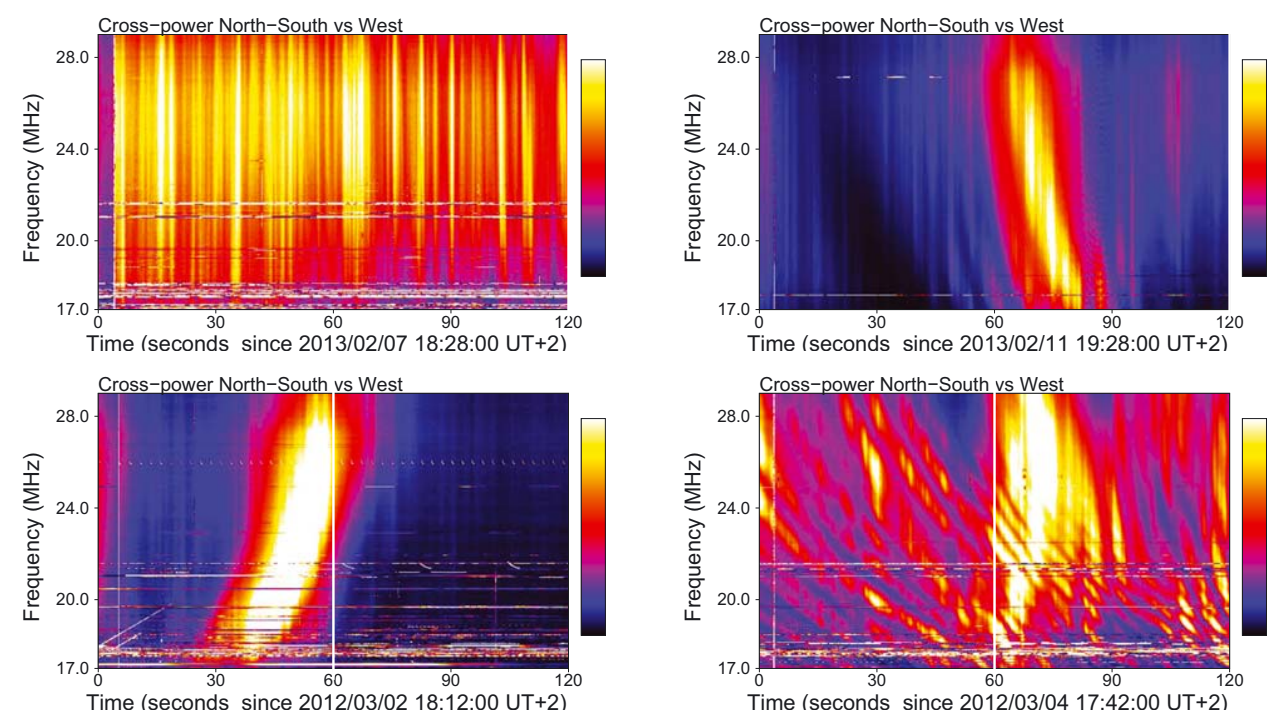

Figure 3: Examples of joint manifestation of interplanetary and ionosphere scintillations on dynamic spectra (radio source 3c144, UTR-2). Interplanetary scintillations are visible as vertical narrow (short) structures; the top-left spectrum is a typical example. Ionosphere scintillations cause slower modulation of spectral density and generate patterns of diverse morphology, including burst-like events. All the visible patterns originate from propagation effects (scintillations). The range of this modulation is $+3 d B \ldots-10 d B$ and more.

and hundreds of pictures. Some other examples of such propagation effects are shown in Figures $5(\mathrm{~B})$ and 8.

\section{Solar system observations}

\subsection{The solar corona}

At low frequencies, the solar corona is a source of sporadic radio emission with very diverse time-frequency power distributions. In spite of high flux, the dynamic spectra may be affected by various propagation effects in the ionosphere of Earth as well as by terrestrial RFI. Reliable identification of such spurious patterns can be achieved only by synchronous observations with radio telescopes separated by distances from hundreds to thousands of kilometers, which ensure de-correlation of RFI and propagation effects.

Figure 4 (A) illustrates the role of "synergy" for discrimination between spurious and bona fide elements on dynamic spectra. Parallel observations made it possible to identify small size (short and narrowband) elements at dynamic spectra as solar radio emission, being neither RFI nor propagation effects. The similar appearance of these elements on two dynamic spectra registered at antennas spaced apart by about 2,000 km is highly unlikely to be caused by RFI or propagation effects, because these negative factors are known to be strongly de-correlated at such distances. For example, a narrow-band RFI visible as a horizontal line in the bottom dynamic spectrum of Figure 4 (A) (NDA) is absent in the top spectrum (UTR-2). 

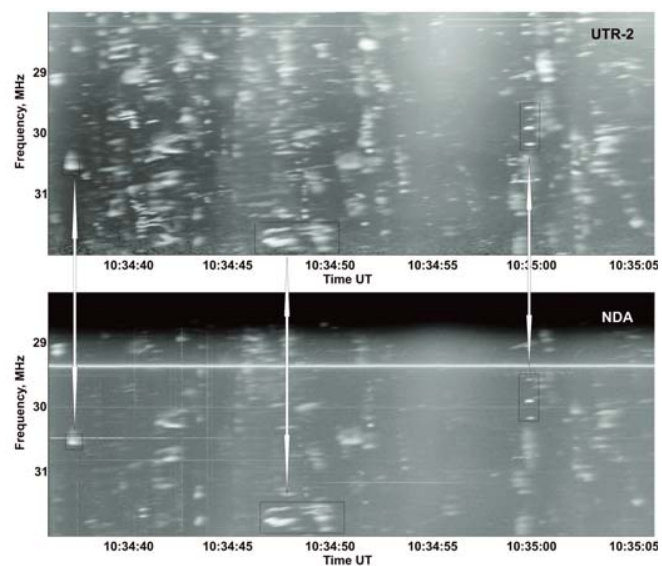

A)

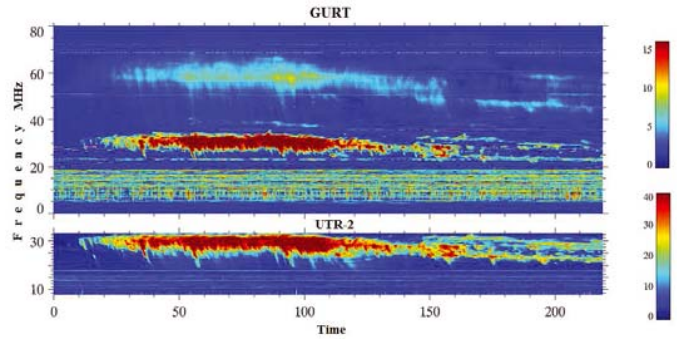

B)

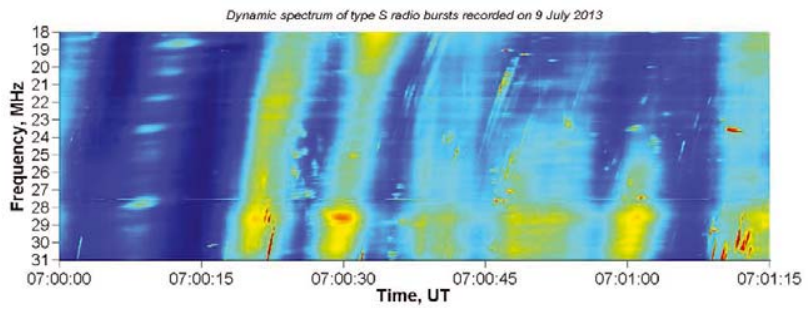

C)

Figure 4: Results of simultaneous observations of solar sporadic decameter radio emission by two radio telescopes. Panel (A): UTR-2 (top) and NDA (bottom) radio telescopes. Panel (B): Type II solar burst observed in parallel by a single sub-array of GURT (top) and UTR-2 (bottom) radio telescopes on 25 July 2014; the start time is 07:11:15 UT. Panel (C): Solar bursts observed by UTR-2 on 9 July 2013 (in parallel with LOFAR, data not shown).

Figure 4 (B) shows an example of synergy between two radio telescopes with overlapping bandwidth (GURT and UTR-2). Although the effective area of a single GURT sub-array is low, its much broader frequency range $(8 \ldots 80 \mathrm{MHz}$ vs $8 \ldots 32 \mathrm{MHz}$ of UTR-2) made it possible to obtain new valuable information: the second harmonic of the solar burst is clearly visible in the top dynamic spectrum.

The dynamic spectrum in Figure $4(\mathrm{C})$, registered by UTR-2, displays solar bursts with super-fine details. Simultaneous observations were also carried out by LOFAR [Morosan et al., 2014]. Joint analysis will be performed in the nearest future. The central question is to decide if particular fine details are real manifestations of solar emission or if they are spurious elements caused by RFI or propagation effects.

\subsection{The solar wind}

Low-frequency radio astronomy provides unique opportunities for the studies of the solar wind, mainly because propagation effects in inhomogeneous interplanetary plasma are maximally pronounced at low frequencies. For example, observations of the solar wind at high elongations, including the space beyond Earth's orbit, are possible mainly at low-frequencies.

To measure parameters of the solar wind, synchronous observations of interplanetary scintillations at several radio telescopes have been routinely carried out for decades. Therefore, the idea of "synergy" between remote radio telescopes fits naturally into this type of studies. 


\subsection{Jupiter}

Decameter emission from Jupiter (and Io) is characterized by rich and diverse structures visible on dynamic spectra. Examples of such spectra registered in multi-antenna observations are shown in Figure 5. Analysis of the spectra brings important information about the underlying mechanisms in the Jovian magnetosphere. In spite of more than six decades of intensive studies, Jovian decameter emission is far from being completely understood, and new observations bring new surprises (see, for example, Figure $5(\mathrm{C})$ ).
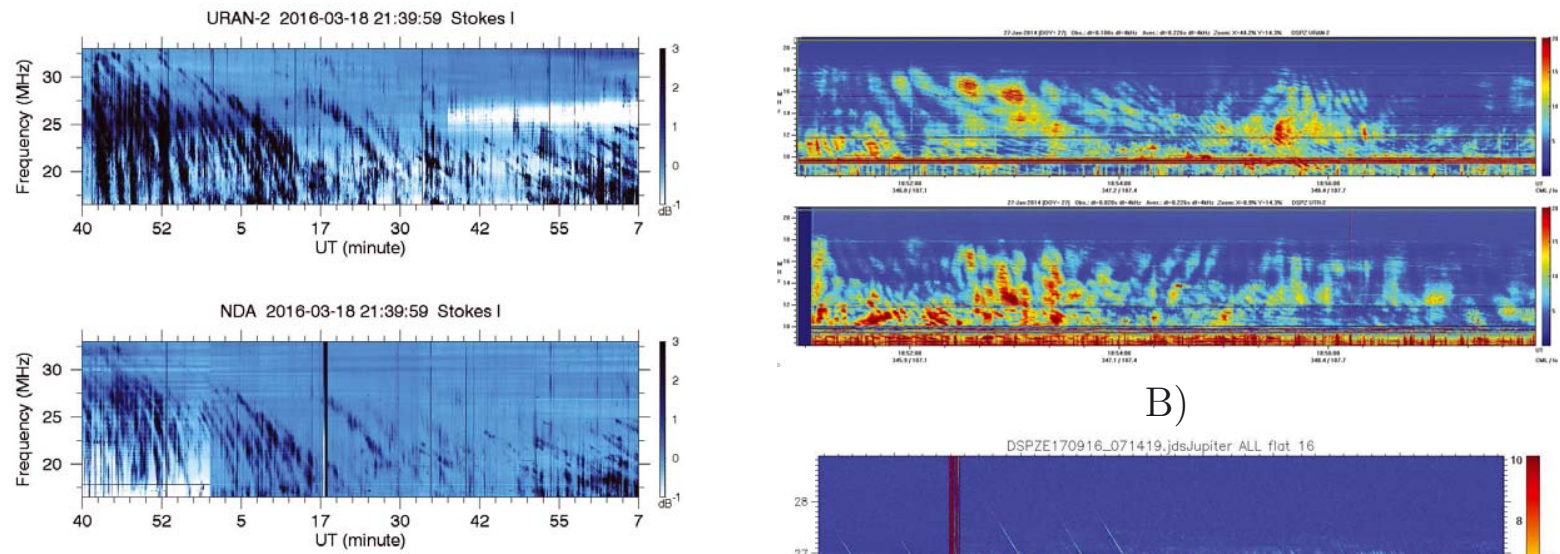

B)

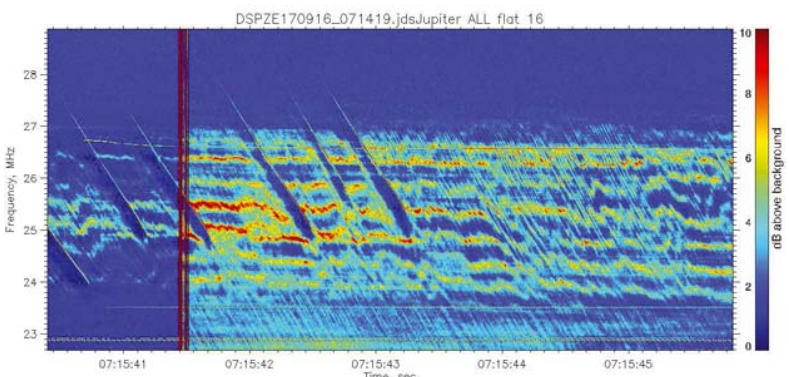

C)

A)

Figure 5: Dynamic spectra of Jovian decameter radio emission simultaneously registered by several radio telescopes. Panel (A): URAN-2 (top), NDA (middle), and UTR-2 (bottom). It is an example of good correspondence between the spectra registered at different radio telescopes. We can be sure that the patterns are of Jovian nature. Panel (B): URAN-2 (top) and UTR2 (bottom). It is an example of radical distinctions between spectra caused by ionosphere scintillations. If a single telescope were used, it would be impossible to discriminate between the bona fide Jovian emission and propagation effects. Moreover, it is likely that the spurious patterns could be falsely ascribed to the Jovian magnetosphere. Panel (C): UTR-2 (in parallel with the Juno mission). An example of interesting but uncertain results obtained in a single site observation. Unusual "absorption bursts" with frequency drift run across the complex zebra pattern. Until Juno data are available, it is not clear whether the effect is of Jovian origin or caused by some nuisance factors.

Scintillations and RFI quite frequently distort the spectra. Simultaneous observations at remote antennas are capable to identify reliably such spurious effects because these effects tend to de-correlate as the distance increases. An example is given in Figure 5 (B).

Another example of simultaneous observations of Jovian decameter emission is described in the paper by Imai et al. [2016]. Three radio telescopes were used in that study: LWA-1 (USA), NDA (France), and URAN-2 (Ukraine). This time the maximal separation was 
about $8,500 \mathrm{~km}$. The study was focused at the beaming structure of Jovian decametric S-bursts.

\subsection{Saturn}

Low-frequency radio emission of Saturn is known to be caused by Saturn Electrostatic Discharges (SED), or lightning. It is strongly associated with the storms observed in the atmosphere of Saturn. In dynamic spectra registered by the UTR-2 radio telescope, this type of emission looks like a series of wide-band short pulses without significant frequency drift, often coalescing and concentrated in groups [Konovalenko et al., 2013] (Figure 6). The pulses are often modulated in a complex pattern in the time-frequency plane.

A crucial question is whether the pulses originate from Saturn lightning or from RFI, either technogenic or natural (terrestrial lightnings). If the signals are really from Saturn, another question is whether the modulation reflects some intrinsic mechanisms in the atmosphere of Saturn or whether it is caused by interplanetary and/or ionospheric scintillations. To answer these questions it would be very useful to complement observations at UTR-2 by dynamic spectra registered in parallel by at least one radio telescope spaced apart from UTR-2 and having a comparable effective area.

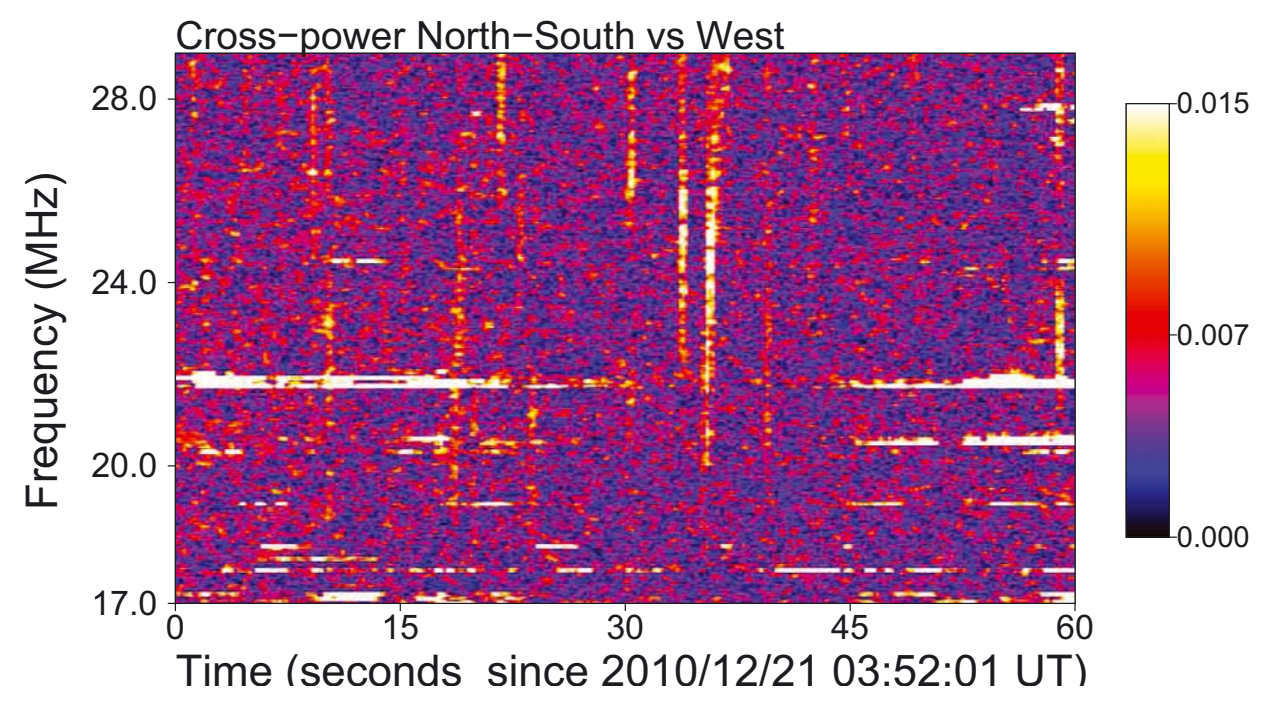

Figure 6: Dynamic spectra of the signals from Saturn electrostatic discharges (lightnings) registered by UTR-2; storm J, December 2010.

\section{Observations of galactic objects}

\subsection{Pulsars}

Individual pulses of pulsars are visible in low-frequency dynamic spectra as short broadband pulses with pronounced quadratic frequency drift towards the low frequencies. The 
magnitude of the drift is proportional to the dispersion measure of the pulsar (Figure 7). An essential feature of such patterns is modulation of the individual pulses in the timefrequency plane. Such modulation may reflect the intrinsic pulse-generating mechanism, but equally reasonably it may be caused by scintillation of the signal in interplanetary and ionospheric plasma. Synchronous observations using at least two radio telescopes are capable to discriminate between these two mechanisms.

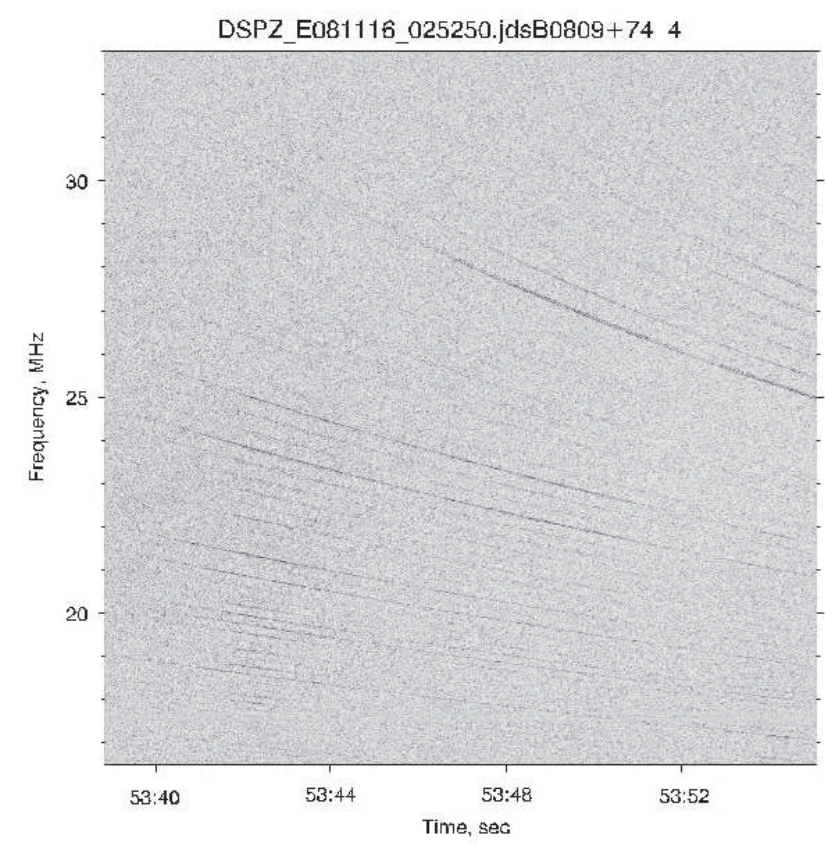

Figure 7: Individual pulses of pulsar PSR B0809+74 observed with the UTR-2 radio telescope on 8 Nov. 2016 at 02:53 UT.

\subsection{Active stars}

Observations of the active stars AD Leonis and EV Lacertae have been performed at the UTR-2 radio telescope for decades. The registration of signals was initially carried out in several narrow spectral bands with ambiguous results. Since 2007 such observations have been carried out on a regular basis using a new generation of back-end facilities allowing registration of dynamic spectra in a wide band of 1-2 octaves (see Table 2). Data processing, analysis and visualization was performed using new tailor-made software oriented to visualization of dynamic spectra with maximal sensitivity. The search was focused on wideband burst-like events and resulted in their apparent detection. Some examples of detected events are shown in the top two panels of Figure 8. Nevertheless, more detailed scrutiny of all the data available raised serious doubts on the real origin of the detected bursts.

To resolve the uncertainty, new series of observations were performed. This time the telescope was aimed not at active stars, but at "null-spots", arbitrarily chosen points at the sky. In spite of the absence of known active stars in the vicinity of the null-spots, quite many burst-like events still were detected in dynamic spectra (see the lower two panels 
in Figure 8). The most likely cause of these events is "dynamic confusion". This effect is caused by weak radio sources within the beam of the radio telescope. Their signals experience scintillation and occasional focusing on inhomogeneities of ionospheric plasma and generate burst-like pattern on dynamic spectra. Examples of such spurious bursts, which are definitely caused by propagation effects, are shown in Figure 3.
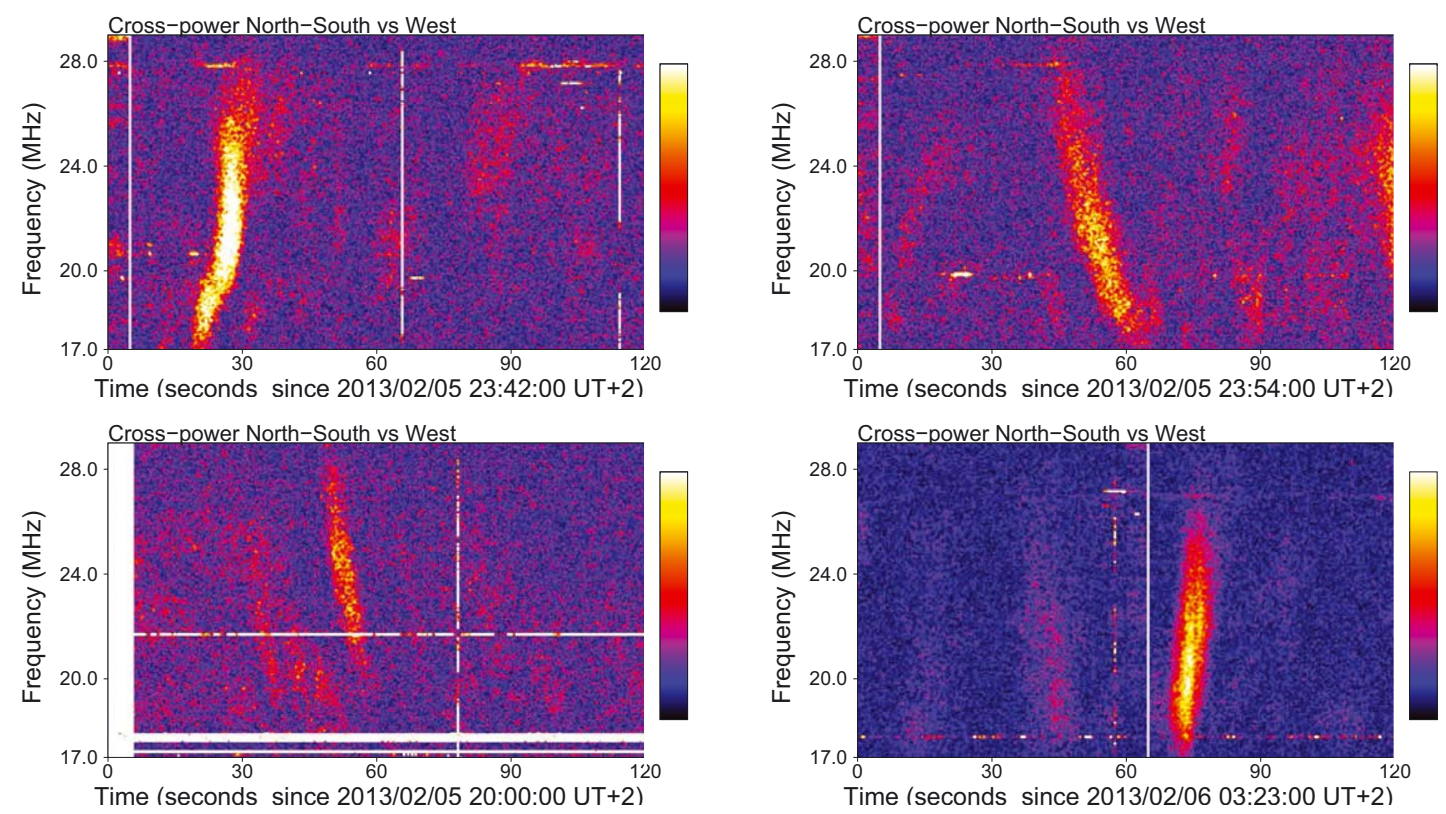

Figure 8: Dynamic spectra registered during observation of the flare star AD Leonis (top) and randomly chosen "null-spots" (bottom) at UTR-2 radio telescope. The burst-like events are highly likely to be caused by a "dynamic confusion" effect, not by sporadic emission from the star itself. The effect originates from ionosphere scintillations and focusing of weak radio sources within the main beam (or nearest side lobes) of the radio telescope. The maximal flux of the bursts corresponds to 1-2\% of the power of the galactic background received by the "NorthSouth" and "West" antennas of UTR-2, which illustrates its high sensitivity.

Another logically possible explanation for our findings is that the actual prevalence of burst-like sporadic radio emission in the universe is much higher than expected, and it is not necessarily associated with known active stars. This could explain the presence of burst-like events on dynamic spectra for null-spots.

Only simultaneous observations with at least two radio telescopes could prove decisively if particular bursts represent real sporadic emission or if it is a manifestation of the "dynamic confusion" effect. This strategy of analysis is equally reasonably applicable to observations aimed at other classes of transient phenomena.

\section{Conclusions}

In this paper, we have shown the necessity of "synergy" between several radio telescopes for solving many crucial problems existing in the low-frequency radio astronomy. Both 
terrestrial and space-borne instruments may be involved in such simultaneous observations. Normally, no mutual coherence of signal registration is required between different radio telescopes. We illustrated the usefulness of synergy by some examples of our real synchronous observations involving Ukrainian (UTR-2, URAN-1 ... URAN-4, GURT) and French (NDA) radio telescopes. This proves that synergistic radio astronomical observations could be quite easily implemented using the existing low-frequency radio telescopes. In this paper we have shown mainly methodological capabilities of multi-antenna observations. Most observational results presented here are new. They are neither published nor analyzed from an astrophysical standpoint. This work is planned to be done in subsequent publications.

Acknowledgments. The Editors thank two anonymous reviewers for their help in evaluating this paper.

\section{References}

Boischot, A., C. Rosolen, M. G. Aubier, G. Daigne, F. Genova, Y. Leblanc, A. Lecacheux, J. de la Noë, and B. M. Pedersen, A new high gain, broadband steerable, array to study Jovian decametric emission, Icarus, 43, 399-407, 1980.

Imai, M., A. Lecacheux, T. E. Clarke, C. A. Higgins, M. Panchenko, J. Dowell, K. Imai, A. I. Brazhenko, A. V. Frantsuzenko, and A. A. Konovalenko, The beaming structures of Jupiter's decametric common S-bursts observed from the LWA1, NDA, and URAN2 radio telescopes, Astrophys. J., 826, id.176, 6 pp., 2016.

Konovalenko, A. A., et al. (14 co-authors), Earliest recorded ground-based decameter wavelength observations of Saturn's lightning during the giant E-storm detected by Cassini spacecraft in early 2006, Icarus, 224, 14-23, 2013.

Konovalenko, A.A., et al. (71 co-authors), The modern radio astronomy network in Ukraine: UTR-2, URAN and GURT, Exp. Astron., 42, 11-48, 2016.

Morosan, D. E., et al. (84 co-authors), LOFAR tied-array imaging of type III solar radio bursts, Astron. Astrophys., 568, id.A67, 8 pp., 2014.

Taylor, G. B., et al. (34 co-authors), First light for the first station of the long wavelength array, J. Astron. Instrum., 1, 1-29, 2012.

van Haarlem, M., et al. (200 co-authors), LOFAR: The LOw-Frequency ARray, Astron. Astrophys., 556, id.A2, 53 pp., 2013.

Zarka, P., J. N. Girard, M. Tagger, and L. Denis, LSS/NenuFAR: The LOFAR super station project in Nançay, SF2A-2012: Proceedings of the Annual meeting of the French Society of Astronomy and Astrophysics, edited by S. Boissier, P. de Laverny, N. Nardetto, R. Samadi, D. Valls-Gabaud, and H. Wozniak, 687-694, 2012. 